\title{
Testing cosmological variability of fundamental constants
}

\author{
D. A. Varshalovich, A. Y. Potekhin, and A. V. Ivanchik \\ Ioffe Physico-Technical Institute, 194021 St. Petersburg, Russia
}

\begin{abstract}
One of the topical problems of contemporary physics is a possible variability of the fundamental constants. Here we consider possible variability of two dimensionless constants which are most important for calculation of atomic and molecular spectra (in particular, the X-ray ones): the fine-structure constant $\alpha=e^{2} / \hbar c$ and the proton-toelectron mass ratio $\mu=m_{p} / m_{e}$. Values of the physical constants in the early epochs are estimated directly from observations of quasars - the most powerful sources of radiation, whose spectra were formed when the Universe was several times younger than now. A critical analysis of the available results leads to the conclusion that present-day data do not reveal any statistically significant evidence for variations of the fundamental constants under study. The most reliable upper limits to possible variation rates at the $95 \%$ confidence level, obtained in our work, read:
\end{abstract}

$$
|\dot{\alpha} / \alpha|<1.4 \times 10^{-14} \mathrm{yr}^{-1}, \quad|\dot{\mu} / \mu|<1.5 \times 10^{-14} \mathrm{yr}^{-1}
$$

on the average over the last $10^{10} \mathrm{yr}$.

\section{INTRODUCTION}

Contemporary theories (SUSY GUT, superstring and others) not only predict the dependence of fundamental physical constants on energy ${ }^{1}$, but also have cosmological solutions in which low-energy values of these constants vary with the cosmological time. The predicted variation at the present epoch is small but nonzero, and it depends on theoretical model. In particular, Damour and Polyakov [1] have developed a modern version of the string theory, whose parameters could be determined from cosmological variations of the coupling constants and hadronto-electron mass ratios. Clearly, a discovery of these variations would be a great step in our understanding of Nature. Even a reliable upper bound on a possible variation rate of a fundamental constant presents a valuable tool for selecting viable theoretical models.

1) The prediction of the theory that the fundamental constants depend on the energy of interaction has been confirmed in experiment. In this paper, we consider only the space-time variability of their low-energy limits. 
Historically, a hypothesis that the fundamental constants may depend on the cosmological time $t$ (that is the age of the Universe) was first discussed by Milne [2] and Dirac [3]. The latter author proposed his famous "large-number hypothesis" and suggested that the gravitational constant was directly proportional to $t$. Later the variability of fundamental constants was analyzed, using different arguments, by Gamow [4], Dyson [5], and others. The interest in the problem has been revived due to recent major achievements in GUT and Superstring models (e.g., [1]).

Presently, the fundamental constants are being measured with a relative error of $\sim 10^{-8}$. These measurements obviously rule out considerable variations of the constants on a short time scale, but do not exclude their changes over the lifetime of the Universe, $\sim 1.5 \times 10^{10}$ years. Moreover, one cannot rule out the possibility that the constants differ in widely separated regions of the Universe; this could be disproved only by astrophysical observations and different kinds of experiments.

Laboratory experiments cannot trace possible variation of a fundamental constant during the entire history of the Universe. Fortunately, Nature has provided us with a tool for direct measuring the physical constants in the early epochs. This tool is based on observations of quasars, the most powerful sources of radiation. Many quasars belong to most distant objects we can observe. Light from the distant quasars travels to us about $10^{10}$ years. This means that the quasar spectra registered now were formed $\sim 10^{10}$ years ago. The wavelengths of the lines ob-

served in these spectra $\left(\lambda_{\text {obs }}\right)$ increase compared to their laboratory values $\left(\lambda_{\text {lab }}\right)$ in proportion $\lambda_{\text {obs }}=\lambda_{\text {lab }}(1+z)$, where the cosmological redshift $z$ can be used to determine the age of the Universe at the line-formation epoch. In some cases, the redshift is as high as $z \sim 3-5$, so that the intrinsically far-ultraviolet lines are registered in the visible range. The examples are demonstrated in Fig. 1. Analysing these spectra we may study the epoch when the Universe was several times younger than now.

Here we review briefly the studies of the space-time variability of the finestructure constant $\alpha$ and the proton-to-electron mass ratio $\mu$.

\section{FINE-STRUCTURE CONSTANT}

Various tests of the fundamental constant variability differ in space-time regions of the Universe which they cover. Local tests relate to the values of constants on the Earth and in the Solar system. In particular, laboratory tests infer the possible variation of certain combinations of constants "here and now" from comparison of different frequency standards. Geophysical tests impose constraints on combinations of fundamental constants over the past history of the Solar system, although most of these constraints are very indirect. In contrast, astrophysical tests allows one to "measure" the values of fundamental constants in distant areas of the early Universe. 

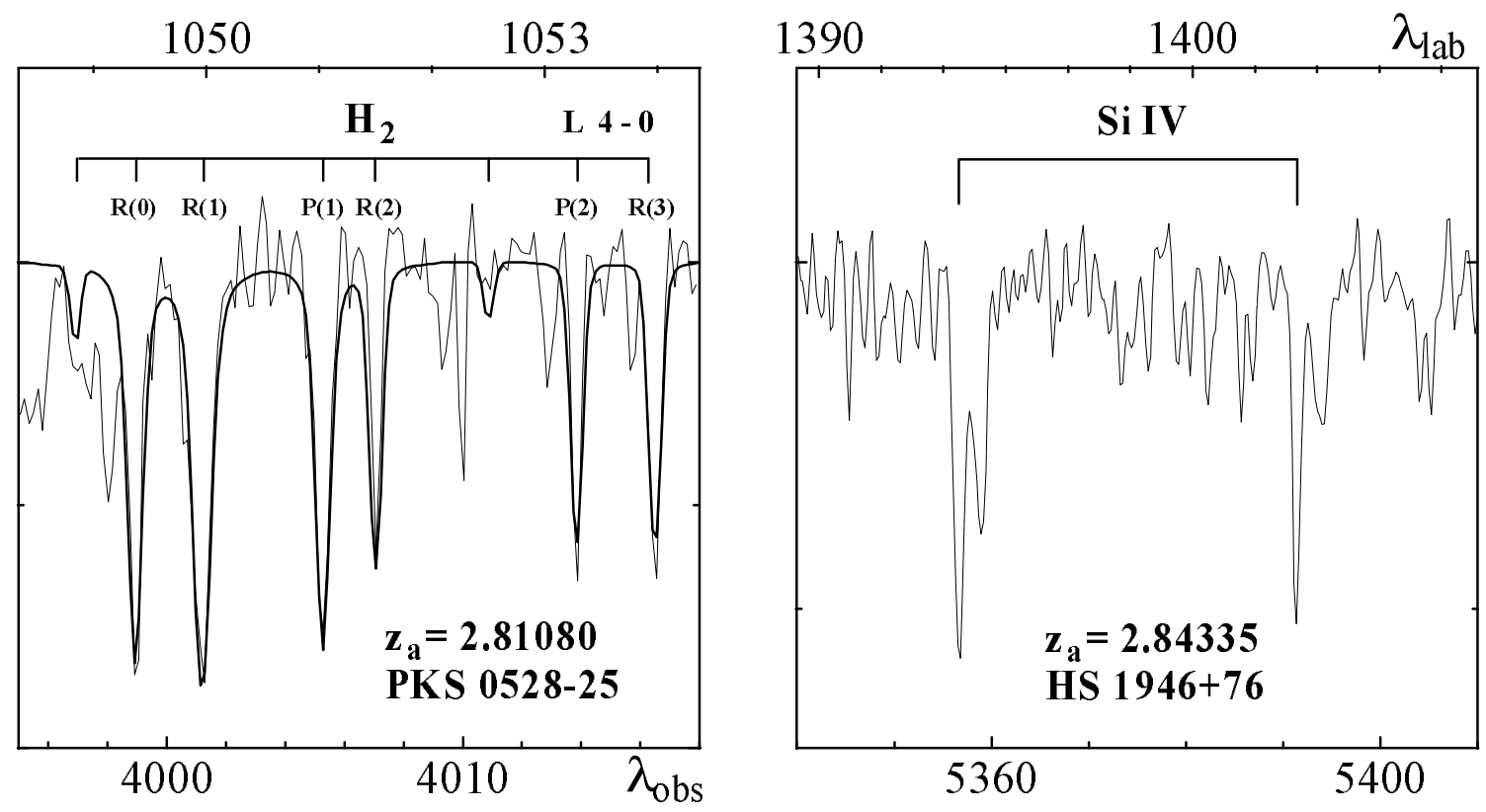

FIGURE 1. Portions of quasar spectra which show the absorption lines of $\mathrm{H}_{2}$ and Si IV with large redshifts, $z=\left(\lambda_{\text {obs }}-\lambda_{\text {lab }}\right) / \lambda_{\text {lab }} \approx 2.8$. The lower horizontal axis gives the wavelengths in the observer's frame $\left(\lambda_{\text {obs }}\right)$ and the upper axis gives the wavelengths in the quasar's frame $\lambda_{\text {lab }}$ (in $\AA$ ). (a) The spectrum (thin line) of the quasar PKS 0528-250, obtained with the 4-meter CTIO telescope (Chile), containing $\mathrm{H}_{2}$ lines which belong to the $\mathrm{L} 4-0$ branch of the spectrum; thick line plots the spectral fit. (b) The spectrum of the quasar HS 1946+76, obtained with the 6-meter SAO telescope (Russia), containing Si IV doublet lines which correspond to the ${ }^{2} \mathrm{~S}_{1 / 2} \rightarrow{ }^{2} \mathrm{P}_{3 / 2}$ and ${ }^{2} \mathrm{~S}_{1 / 2} \rightarrow{ }^{2} \mathrm{P}_{1 / 2}$ transitions.

\section{Local tests}

\section{Laboratory experiments}

There were a number of laboratory experimets aimed at detection of trends of the fundamental constants with time by comparison of frequency standards which have different dependences on the constants. We mention only two of the published experiments.

Comparison of $\mathrm{H}$-masers with Cs-clocks during 427 days revealed a relative $(\mathrm{H}-$ $\mathrm{Cs}$ ) frequency drift with a rate $1.5 \times 10^{-16}$ per day, while the rates of $(\mathrm{H}-\mathrm{H})$ and (Cs-Cs) drifts (i.e., the drifts between identical standards, used to control their stability) were less than $1 \times 10^{-16}$ per day [6]. A similar result was found in comparison of a $\mathrm{Hg}^{+}$-clock with a $\mathrm{H}$-maser during 140 days [7]: the rate of the relative frequency drift was less than $(2 \pm 1) \times 10^{-16}$ per day.

Such a drift is treated as a consequence of a difference in the long-term stability 
of different atomic clocks. In principle, however, it may be caused by variation of $\alpha$. That is why it gives an upper limit to the $\alpha$ variation [7]: $|\dot{\alpha} / \alpha| \leq 3.7 \times 10^{-14} \mathrm{yr}^{-1}$.

\section{Geophysical tests}

The strongest bound on the possible time-variation rate of $\alpha$ was derived in 1976 by Shlyakhter [8], and recently, from a more detailed analysis, by Damour and Dyson [9], who obtained $|\dot{\alpha} / \alpha|<0.7 \times 10^{-16} \mathrm{yr}^{-1}$, The analysis was based on measurements of isotope ratios in the Oklo site in Gabon, where a unique natural uranium nuclear fission reactor had operated 1.8 billion years ago. The isotope ratios of samarium produced in this reactor by the neutron capture reaction ${ }^{149} \mathrm{Sm}+n \rightarrow{ }^{150} \mathrm{Sm}+\gamma$ would be completely different, if the energy of the nuclear resonance responsible for this capture were shifted at least by $0.1 \mathrm{eV}$.

Another strong bound, $|\dot{\alpha} / \alpha|<5 \times 10^{-15} \mathrm{yr}^{-1}$, was obtained by Dyson [5] from an isotopic analysis of natural radioactive decay products in meteorites.

A weak point of these tests is their dependence on the model of the phenomenon, fairly complex, involving many physical effects. For instance, Damour and Dyson [9] estimated possible shift of the above-mentioned resonance due to the $\alpha$ variation, assuming that the Coulomb energy of the excited state of ${ }^{150} \mathrm{Sm}^{*}$, responsible for the resonance, is not less than the Coulomb energy of the ground state of ${ }^{150} \mathrm{Sm}$. In absence of experimental data on the nuclear state in question, this assumption is not justified, since heavy excited nuclei often have Coulomb energies smaller than those for their ground states [10]. Furthermore, a correlation between the constants of strong and electroweak interactions (which is likely in the frame of modern theory) might lead to further softening of the mentioned bounds by 100fold, to $|\dot{\alpha} / \alpha|<5 \times 10^{-15} \mathrm{yr}^{-1}$, as noted by Sisterna and Vucetich [11].

In addition, the local tests cannot be extended to distant space regions and to the early Universe, since the law of possible space-time variation of $\alpha$ is unknown a priory. It is the extragalactic astronomy that allows us to study these remote regions of spacetime, in particular the regions which were causally disconnected at the epoch of formation of the observed absorption spectra.

\section{Astrophysical tests}

To find out whether $\alpha$ changed over the cosmological time, we have studied the fine splitting of the doublet lines of Si IV, C IV, Mg II and other ions, observed in the spectra of distant quasars. According to quantum electrodynamics, the relative splitting of these lines $\delta \lambda / \lambda$ is proportional to $\alpha^{2}$ (neglecting very small corrections). Consequently, if $\alpha$ changed with time, then $\delta \lambda / \lambda$ would depend on the cosmological redshift $z$. This method of measuring $\alpha$ in distant regions of the Universe had been first suggested by Savedoff [12] and was used later by other authors. For instance, Wolfe et al. [13] derived an estimate $|\dot{\alpha} / \alpha|<4 \times 10^{-12} \mathrm{yr}^{-1}$ from an observation of the Mg II absorption doublet at $z=0.524$. 
TABLE 1. Variation of $\alpha$ value estimated from redshifted Si IV fine-splitting doublets.

\begin{tabular}{lcrc}
\hline Quasar & $z$ & $\Delta \alpha / \alpha$ & Ref. \\
\hline HS 1946+76 & 3.050079 & 1.58 & {$[16]$} \\
HS 1946+76 & 3.049312 & 0.34 & {$[16]$} \\
HS 1946+76 & 2.843357 & 0.59 & {$[16]$} \\
S4 0636+76 & 2.904528 & 1.37 & {$[16]$} \\
S5 0014+81 & 2.801356 & -1.80 & {$[16]$} \\
S5 0014+81 & 2.800840 & -1.70 & {$[16]$} \\
S5 0014+81 & 2.800030 & 1.11 & {$[16]$} \\
& & & \\
PKS 0424-13 & 2.100027 & -4.51 & {$[15]$} \\
Q 0450-13 & 2.230199 & -1.48 & {$[15]$} \\
Q 0450-13 & 2.104986 & 0.02 & {$[15]$} \\
Q 0450-13 & 2.066646 & 1.03 & {$[15]$} \\
& & & \\
J 2233-60 & 1.867484 & -1.92 & {$[17]$} \\
J 2233-60 & 1.869756 & -2.21 & {$[17]$} \\
J 2233-60 & 1.871074 & -1.41 & {$[17]$} \\
J 2233-60 & 1.925971 & 1.11 & {$[17]$} \\
J 2233-60 & 1.941979 & 0.48 & {$[17]$} \\
\hline
\end{tabular}

An approximate formula which relates a deviation of $\alpha$ at redshift $z$ from its current value, $\Delta \alpha_{z}$, with measured $\delta \lambda / \lambda$ in the extragalactic spectra and in laboratory reads

$$
\Delta \alpha_{z} \approx \frac{c_{r}}{2}\left[\frac{(\delta \lambda / \lambda)_{z}}{(\delta \lambda / \lambda)_{0}}-1\right],
$$

where $c_{r} \sim 1$ takes into account radiation corrections [14]: for instance, for Si IV $c_{r} \approx 0.9$.

Many high-quality quasar spectra measured in the last decade have enabled us to significantly increase the accuracy of determination of $\delta \lambda / \lambda$ at large $z$. An example of the spectra observed is shown in Fig. 1. For the present report, we have selected the results of high-resolution observations [15-17], most suitable for an analysis of $\alpha$ variation. The values of $\Delta \alpha / \alpha$ calculated from these data according to Eq. (1) are given in Table 1.

As a result, we obtain a new estimate of the possible deviation of the finestructure constant at $z=2-4$ from its present $(z=0)$ value:

$$
\Delta \alpha / \alpha=(-4.6 \pm 4.3[\text { stat }] \pm 1.4[\text { syst }]) \times 10^{-5},
$$

where the statistical error is obtained from the scatter of astronomical data (at large $z$ ) and the systematic one is estimated from the uncertainty of the fine splitting measurement in the laboratory $[18,19]$ (at $z=0$, which serves as the reference point for the estimation of $\Delta \alpha$ ). The corresponding upper limit of the $\alpha$ variation rate averaged over $\sim 10^{10} \mathrm{yr}$ is 


$$
|\dot{\alpha} / \alpha|<1.4 \times 10^{-14} \mathrm{yr}^{-1}
$$

(at the $95 \%$ confidence level). This constraint is much more stringent than those obtained from all but one previous astronomical observations. The notable exception is presented by Webb et al. [20], who have analysed spectroscopic data of similar quality, but estimated $\alpha$ from comparison of Fe II and Mg II fine-splitted walelengths in extragalactic spectra and in the laboratory. Their result indicates a tentative time-variation of $\alpha: \Delta \alpha / \alpha=(-1.9 \pm 0.5) \times 10^{-5}$ at $z=1.0-1.6$. Note, however, two important sources of a possible systematic error which could mimic

the effect: (a) Fe,II and Mg II lines used are situated in different orders of the echelle-spectra, so relative shifts in calibration of the different orders can simulate the effect of $\alpha$-variation, and (b) were the relative abundances of $\mathrm{Mg}$ isotopes changing during the cosmological evolution, the Mg II lines would be subjected to an additional $z$-dependent shift relative to the Fe II lines, quite sufficient to simulate the variation of $\alpha$ (this shift can be easily estimated from recent laboratory measurements [21]). In contrast, the method based on the fine splitting of a line of the same ion species ( $\mathrm{Si}$ IV in the above example) is not affected by these two uncertainty sources. Thus we believe that the restriction (3) is the most reliable at present for the long-term history of the Universe.

According to our analysis, some theoretical models are inconsistent with observations. For example, power laws $\alpha \propto t^{n}$ with $n=1,-1 / 4$, and $-4 / 3$, published by various authors in 1980s, are excluded. Moreover, the Teller-Dyson's hypothesis on the logarithmic dependence of $\alpha$ on $t[22,5]$ has also been shown to be inconsistent with observations.

Many regions of formation of the spectral lines, observed at large redshifts in different directions in the sky, had been causally disconnected at the epochs of line formation. Thus, no information could have been exchanged between these regions of the Universe and, in principle, the fundamental constants could be different there. However, a separate analysis [23] has shown that $\alpha$ value is the same in different directions in the sky within the $3 \sigma$ relative error $|\Delta \alpha / \alpha|<3 \times 10^{-4}$.

\section{PROTON-TO-ELECTRON MASS RATIO}

The dimensionless constant $\mu=m_{\mathrm{p}} / m_{\mathrm{e}}$ approximately equals the ratio of the constants of strong interaction $g^{2} /(\hbar c) \sim 14$ and electromagnetic interaction $\alpha \approx$ $1 / 137.036$, where $g$ is the effective coupling constant calculated from the amplitude of nucleon- $\pi$-meson scattering at low energy.

In order to check the cosmological variability of $\mu$ we have used high-redshift absorption lines of molecular hydrogen $\mathrm{H}_{2}$ in the spectrum of the quasar $\mathrm{PKS}$ 0528-250. This is the first (and, in a sense, unique) high-redshift system of $\mathrm{H}_{2}$ absorption lines discovered in 1985 [24]. A study of these objects yields information of paramount importance on the physical conditions $\sim 10^{10}$ years ago.

A possibility of distinguishing between the cosmological redshift of spectral wavelengths and shifts due to a variation of $\mu$ arises from the fact that the electronic, 
TABLE 2. Comparison of wavelengths of electron-vibro-rotational lines for $\mathrm{H}_{2}, \mathrm{D}_{2}$, and $T_{2}$.

\begin{tabular}{crrrr}
\hline$i$ & $\lambda_{i}\left(\mathrm{H}_{2}\right)$ & $\lambda_{i}\left(\mathrm{D}_{2}\right)$ & $\lambda_{i}\left(\mathrm{~T}_{2}\right)$ & $K_{i}$ \\
\hline L 0-0 R(1) & 1108.633 & 1103.351 & 1101.021 & $-8.18 \times 10^{-3}$ \\
L 0-2 R(1) & 1077.697 & 1081.153 & 1082.760 & $+5.35 \times 10^{-3}$ \\
L 0-9 R(1) & 992.013 & 1015.610 & 1027.218 & $+3.80 \times 10^{-2}$ \\
\hline
\end{tabular}

vibrational, and rotational energies of $\mathrm{H}_{2}$ each undergo a different dependence on the reduced mass of the molecule. Hence comparing ratios of wavelengths $\lambda_{i}$ of various $\mathrm{H}_{2}$ electron-vibration-rotational lines in a quasar spectrum at some redshift $z$ and in laboratory (at $z=0$ ), we can trace variation of $\mu$. The method had been used previously by Foltz et al. [25], whose analysis was corrected later in our papers $[26,23,27]$. In the latter papers, we calculated the sensitivity coefficients $K_{i}$ of the wavelengths $\lambda_{i}$ with respect to possible variation of $\mu$ and applied a linear regression analysis to the measured redshifts of individual lines $z_{i}$ as function of $K_{i}$. An illustration of the wavelength dependences on the mass of the nucleus is given in Table 2, where a few resonance wavelengths of hydrogen, deuterium, and tritium molecules are listed. One can see that, as the nuclear mass increases, different wavelengths shift in different directions. More complete tables, as well as two algorithms of $K_{i}$ calculation, are given in Refs. [23,28].

Thus, if the proton mass in the epoch of line formation were different from the present value, the measured $z_{i}$ and $K_{i}$ values would correlate:

$$
\frac{z_{i}}{z_{k}}=\frac{\left(\lambda_{i} / \lambda_{k}\right)_{z}}{\left(\lambda_{i} / \lambda_{k}\right)_{0}} \simeq 1+\left(K_{i}-K_{k}\right)\left(\frac{\Delta \mu}{\mu}\right) .
$$

We have performed a $z$-to- $K$ regression analysis using a modern high-resolution spectrum of PKS 0528-250 [28]. Several tens of the $\mathrm{H}_{2}$ lines have been identified; a portion of the spectrum which reveales some of the lines is shown in Fig. 1. The redshift estimates for individual absorption lines with their individual errorbars are plotted in Fig. 2 against their sensitivity coefficients. The resulting parameter estimate and $1 \sigma$ uncertainty is

$$
\Delta \mu / \mu=(-11.5 \pm 7.6[\text { stat }] \pm 1.9[\text { syst }]) \times 10^{-5} .
$$

The $2 \sigma$ confidence interval to $\Delta \mu / \mu$ is

$$
|\Delta \mu / \mu|<2.0 \times 10^{-4} \text {. }
$$

Assuming that the age of the Universe is $\sim 15$ Gyr the redshift of the $\mathrm{H}_{2}$ absorption system $z=2.81080$ corresponds to the elapsed time of 13 Gyr (in the standard cosmological model). Therefore we arrive at the restriction

$$
|\dot{\mu} / \mu|<1.5 \times 10^{-14} \mathrm{yr}^{-1}
$$

on the variation rate of $\mu$, averaged over $90 \%$ of the lifetime of the Universe. 


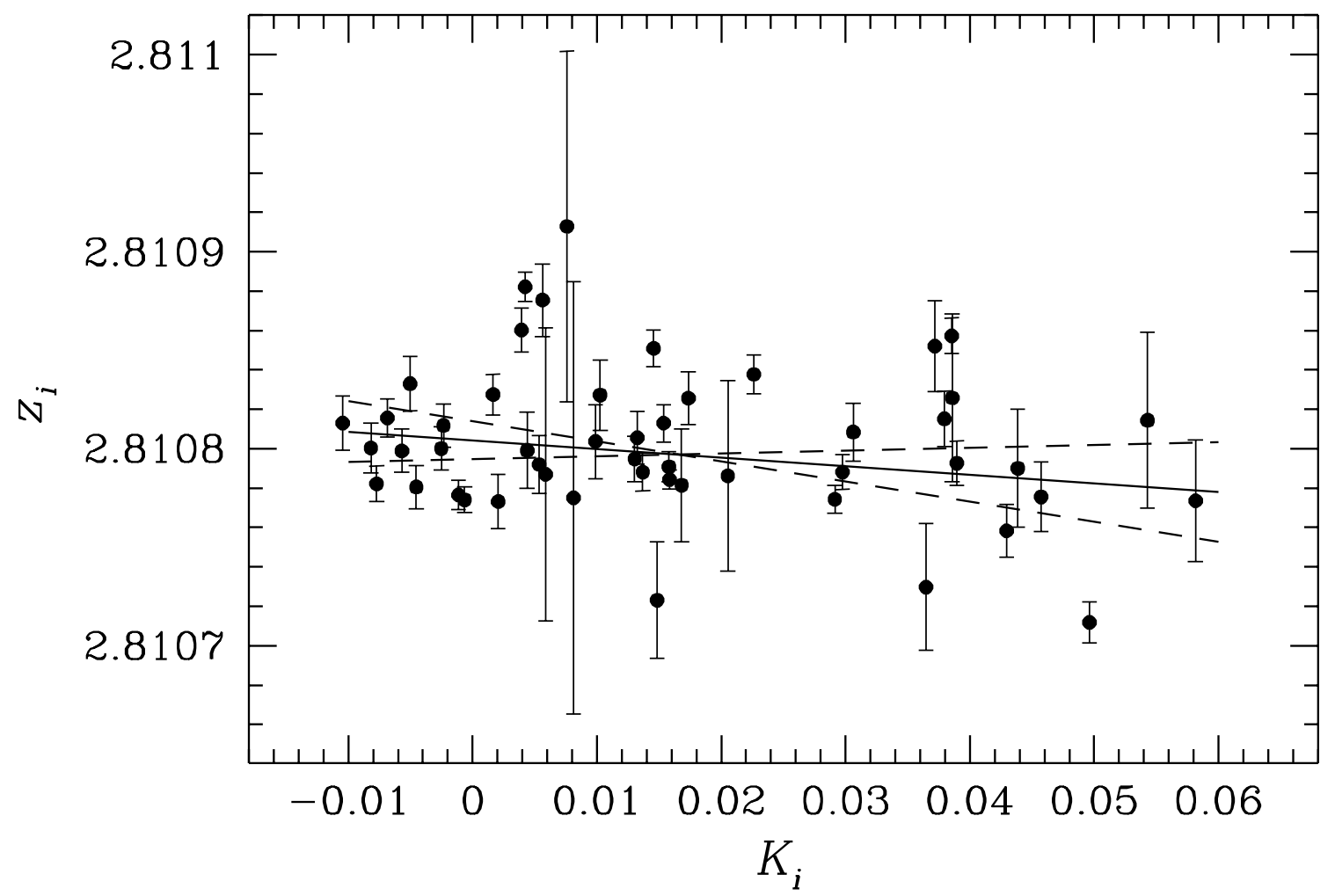

FIGURE 2. Redshift values inferred from an analysis of separate spectral features in an $\mathrm{H}_{2}$ absorption system in the spectrum of the quasar PKS 0528-250, plotted vs. $\lambda_{i}(\mu)$-sensitivity coefficients $K_{i}$. The slanted solid line shows the most probable regression and the dashed ones corespond to $\pm 1 \sigma$ deviations of the slope.

\section{CONCLUSIONS}

Despite the theoretical prediction of the time-dependences of fundamental constants, a statistically significant variation of any of the constants have not been reliably detected up to date, according to our point of view substantiated above. The upper limits obtained indicate that the constants of electroweak and strong interactions did not significantly change over the last $90 \%$ of the history of the Universe. This shows that more precise measurements and observations and their accurate statistical analyses are required in order to detect the expected variations of the fundamental constants.

Acknowledgements. This work was performed in frames of the Project 1.25 of the Russian State Program "Fundamental Metrology" and supported by the grant RFBR 99-02-18232. 


\section{REFERENCES}

1. Damour, T., and Polyakov, A.M., Nucl. Phys. B 423, 532 (1994).

2. Milne, E., Proc. R. Soc. A 158, 324 (1937).

3. Dirac, P.A.M., Nature 139, 323 (1937).

4. Gamow, G., Phys. Rev. Lett. 19, 759 (1967).

5. Dyson, F.J., in Aspects of Quantum Theory, edited by A. Salam and E. P. Wigner, , p. 213 (Cambridge Univ., Cambridge, 1972).

6. Demidov, N.A., Ezhov, E.M., Sakharov, B.A., et al., in Proc. of 6th European Frequency and Time Forum, p. 409 (1992).

7. Prestage, J.D., Tjoelker, R.L., and Maleki, L., Phys. Rev. Lett. 14, 3511 (1995).

8. Shlyakhter, A.I., Nature 25, 340 (1976).

9. Damour, T., and Dyson, F.J., Nucl. Phys. B 480, 37 (1996).

10. Kalvius, G.M., and Shenoy, G.K., Atomic and Nuclear Data Tables 14, 639 (1974).

11. Sisterna, P.D., and Vucetich, H., Phys. Rev. D 41, 1034 (1990).

12. Savedoff, M.P., Nature 264, 340 (1956).

13. Wolfe, A.M., Brown, R.L., and Roberts, M.S., Phys. Rev. Lett 37, 179 (1976).

14. Dzuba, V.A., Flambaum, V.V., and Webb, J.K., Phys. Rev. A, submitted (e-print: physics/9808021).

15. Petitjean, P., Rauch, M., and Carswell, R.F., Astron. Astrohys. 91, 29 (1994).

16. Varshalovich, D.A., Panchuk, V.E., and Ivanchik, A.V., Pis'ma Astron. Zh. (Engl. transl.: Astron. Lett.) 22, 8 (1996).

17. Outram, P.J., Boyle, B.J., Carswell, R.F., Hewett, P.C., and Williams, R.E., Mon. Not. Roy. Astron. Soc. 305, 685 (1999).

18. Morton, D.C., Astrophys. J. Suppl. 77, 119 (1991).; (E) 81, 883 (1992)

19. Kelly, R.L., J. Phys. Chem. Ref. Data NBS 16, Suppl. 1 (1987).

20. Webb, J.K., Flambaum, V.V., Churchill, C.W., Drinkwater, M.J., and Barrow, J.D., Phys. Rev. Lett. 82, 884 (1999).

21. Pickering, J.C., Thorne, A.P., and Webb, J.K, Monthly Not. R. Astron. Soc. 300, 131 (1998).

22. Teller, E., Phys. Rev. 73, 801 (1948).

23. Varshalovich, D.A. and Potekhin, A.Y., Space Sci. Rev. 74, 259 (1995).

24. Levshakov, S.A., and Varshalovich, D.A., Monthly Not. R. Astron. Soc. 212, 517 (1985).

25. Foltz, C.B., Chaffee, F.H., and Black, J.H., Astrophys. J. 324, 267 (1988).

26. Varshalovich, D.A. and Levshakov, S.A., JETP Lett. 58, 237 (1993).

27. Varshalovich, D.A., and Potekhin, A.Y., Pis'ma Astron. Zh. (Engl. transl.: Astron. Lett.) 22, 3 (1996).

28. Potekhin, A.Y., Ivanchik, A.V., Varshalovich, D.A., Lanzetta, K.M., et al., Astrophys. J. 505, 523 (1998). 